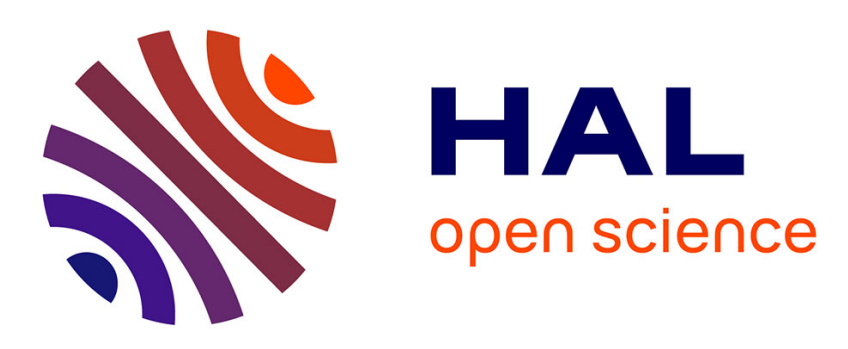

\title{
Manipulation of Autophagy by Bacterial Pathogens Impacts Host Immunity
}

Tobias Kunz, Flávia Viana, Carmen Buchrieser, Pedro Escoll

\section{To cite this version:}

Tobias Kunz, Flávia Viana, Carmen Buchrieser, Pedro Escoll. Manipulation of Autophagy by Bacterial Pathogens Impacts Host Immunity. Pedro Escoll. Bacterial Evasion of the Host Immune System, 25 (4), Caister Academic Press, pp.81-98, 2017, Bacterial Evasion of the Host Immune System, 978-1910190-69-2 (book), 978-1-910190-70-8 (ebook). 10.21775/cimb.025.081 . pasteur-02887892

\section{HAL Id: pasteur-02887892}

https://hal-pasteur.archives-ouvertes.fr/pasteur-02887892

Submitted on 2 Jul 2020

HAL is a multi-disciplinary open access archive for the deposit and dissemination of scientific research documents, whether they are published or not. The documents may come from teaching and research institutions in France or abroad, or from public or private research centers.
L'archive ouverte pluridisciplinaire HAL, est destinée au dépôt et à la diffusion de documents scientifiques de niveau recherche, publiés ou non, émanant des établissements d'enseignement et de recherche français ou étrangers, des laboratoires publics ou privés. 
2

5

6

7

8 9

10

11

12

13

14

15

16

17

18

19

20

21

22

23

24

25

26

27

28

29

30

\title{
Manipulation of autophagy by bacterial pathogens impacts host immunity
}

\author{
Tobias Kunz, Flávia Viana, Carmen Buchrieser ${ }^{*} \&$ Pedro Escoll $^{*}$
}

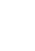

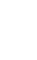

Institut Pasteur, Biologie des Bactéries Intracellulaires, Paris, France and CNRS UMR 3525, Paris, France

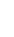

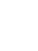

.

5

17

8

9

0

3 *For correspondence: Carmen Buchrieser and Pedro Escoll

Biologie des Bactéries Intracellulaires

Institut Pasteur

28, rue du Dr. Roux, 75724 Paris Cedex 15, France

7 Tel: (33-1)-45-68-83-72

Fax: (33-1)-45-68-87-86

E-mail: chuch@pasteur.fr and pescoll@pasteur.fr

30 
32 Autophagy is a highly conserved catabolic process, degrading unnecessary or damaged 33 components in the eukaryotic cell to maintain cellular homeostasis, but it is also an intrinsic 34 cellular defence mechanism to remove invading pathogens. A crosstalk between autophagy 35 and innate or adaptive immune responses has been recently reported, whereby autophagy 36 influences both, innate and adaptive immunity like the production and secretion of pro37 inflammatory cytokines or MHC class II antigen presentation to T cells. Pathogenic bacteria 38 have evolved diverse strategies to manipulate autophagy, mechanisms and thus also impact 39 host immune responses at different levels. Here we discuss the influence of autophagy on 40 self-autonomous, innate and adaptive immunity and then focus on how bacterial mechanisms 41 that shape autophagy may impact the host immune system. 
43 Maintaining cellular homeostasis requires a coordinated control of metabolic pathways. Thus the cell needs anabolic processes to build new components, and catabolic processes to discard long-lived or damaged components. Autophagy is one of these catabolic processes that leads to lysosomal degradation of unnecessary or damaged proteins, lipids and organelles (Ohsumi, 2014; Rubinsztein et al., 2012). The term "autophagy" describes mainly three different processes: macroautophagy, microautophagy and chaperon-mediated autophagy. In this review we will focus on macroautophagy (hereafter called autophagy), which is characterized by the sequestration of cargo into double-membrane vesicles called autophagosomes (Miller and Celli, 2016).

Autophagy can be divided into selective and non-selective autophagy: in non-selective autophagy a random portion of the cytoplasm is engulfed to degrade long-lived components or to provide nutrients during starvation, while selective autophagy targets selected components for degradation. This includes organelle-specific autophagy, such as removal of mitochondria called mitophagy, and xenophagy which is the removal of invading bacteria (Deretic et al., 2013; Huang and Brumell, 2014).

Intracellular bacteria grow and replicate inside host cells. These bacteria can be divided into two groups: those that replicate in the cytosol, such as Listeria or Shigella, and those that replicate in membrane-derived pseudo-organelles called pathogen-containing vacuoles (PCVs), such as Legionella, Mycobacteria or Salmonella. PCVs facilitate the replication, survival and dormancy of intracellular bacteria. For their formation bacteria exploit the host's membrane system, in particular the dynamic machineries involved in exo/endocytic traffic and autophagy. To enhance survival and proliferation in PCVs, bacteria secrete effector proteins that inhibit their degradation in lysosomes and facilitate the acquisition of membrane sources and nutrients (Ray et al., 2009). In order to ensure survival, intracellular bacteria also need to counteract self-defense mechanisms of the host cell. In animals, cellular self-defense (also known as cell-autonomous immunity) synergizes with the whole-body protection provided by "conventional" immunity (innate and adaptative immunity) to grant resistance to pathogens. While professional immune cells patrol the body in search of pathogens, cellautonomous immunity guards both individual immune and non-immune cells against the immediate threat of infection. The current paradigm shows that cell-autonomous immunity against intracellular pathogens is based on the tight and continuous control of the composition and behaviour of distinct cellular compartments, such as the cytosol, organelles, endosomes, 
autophagosomes and lysosomes (Randow et al., 2013). As the autophagy machinery removes intracellular pathogens by directing them to lysosomal degradation, xenophagy has emerged as an important cellular self-defense process to protect host cells against intracellular bacteria. In this case, engulfment of bacteria by autophagosomes triggers an integrated response within the infected cell, leading to pathogen elimination while professional immune cells are warned about the threat.

As autophagy is an important cellular self-defense mechanism, bacteria evolved various mechanisms to modulate autophagy such as inhibiting autophagosome formation, selfmasking with host proteins to avoid recognition, escaping targeting to autophagosomes or blocking fusion of autophagosomes with lysosomes ( Huang and Brumell, 2014; Escoll et al., 2016). As autophagy is linked to immune responses, bacteria-induced modulation of autophagy impacts also innate and adaptive immune responses (Kuballa et al., 2012).

Here, we will first discuss the general knowledge about autophagosome formation, elongation and maturation, the crosstalk between autophagy and immunity and then focus on the mechanisms used by bacterial pathogens to manipulate autophagy and how these autophagy subversions by bacteria impact the host immune response.

\section{Autophagosome formation, elongation and maturation is a complex process involving conserved host factors}

The pathway leading to autophagy includes signal induction, nucleation and elongation of the phagophore to form the autophagosome, closure of the autophagosome and fusion with lysosomes (Parzych and Klionsky, 2014). More than 35 Autophagy-related proteins (ATGproteins) that have metazoan orthologues have been described in yeast to coordinate autophagosome formation and maturation (Ohsumi, 2014) (Figure 1).

Autophagy induction can be triggered by different signals ranging from nutrient limitation (non-specific autophagy) to the recognition of pathogens or damaged cellular components (Deretic et al., 2013). Most signals for the initiation of autophagy converge through the functions of the mammalian target of rapamycin complex 1 (mTORC1). Under nutrient-rich conditions the mTORC1 complex phosphorylates Unc-51-like kinase 1 (ULK1) and ATG13, which together with the FAK family kinase-interacting protein of 200kDa (FIP200) form the ULK1 complex. When mTORC1 phosphorylates ULK1 and ATG13, the complex cannot be recruited to the phagophore assembly site (PAS), thus inhibiting autophagosome formation (Ganley et al., 2009; Mizushima, 2010). 
Under starvation or stress, mTORC1 becomes inactive and thus its inhibitory action on the ULK1 complex ceases which in turn now phosphorylates FIP200 rendering the complex active (Ganley et al., 2009). The activated ULK1 complex then translocates to the PAS and recruits other proteins such as beclin1 (BECN1) or Atg6 named in yeast, ATG14-like protein

111 (ATG14L) and phosphoinositide 3-kinase regulatory subunit 4 (PIK3R4; vacuolar protein 112 sorting associated $15 \mathrm{Vps} 15$ in yeast). Initiation of phagophore nucleation also results in 113 engagement of PI3-kinase class III (PI3KC3; Vps34 in yeast), which produces PI3P at the 114 phagophore and leads to the recruitment of WD-repeat domain phosphoinositide-interacting 1 115 (WIPI1; Atg18 in yeast) and WIPI2 (Atg21 in yeast). Like a cascade, these proteins recruit 116 further downstream ATG proteins that aid phagophore assembly (Nair et al., 2010; Polson et 117 al., 2014). The PAS needs a source of membranes to form the phagophore-expanding 118 autophagosome. It has been shown that these are mainly mitochondrial-associated ERmembranes (MAMs) (Hamasaki et al., 2013), although several other membranes have also been proposed as sites for nucleation of the phagophore, such as plasma membrane-derived vesicles, mitochondria and the Golgi apparatus (Tooze and Yoshimori, 2010).

Phagophore elongation is then mediated by two ubiquitination-like conjugation systems of ATG proteins. First, ATG7 (E1-like protein) and ATG10 (E2-like protein) conjugate ATG12 124 and ATG5, promoting phagophore elongation (Mizushima et al., 1998; Shintani et al., 1999). ATG12-ATG5 forms a complex with ATG16L1, which associates with the expanding

126 phagophore membrane and gets released after the autophagosome is formed (Mizushima et al., 127 2001; 2003). The ATG12-ATG5-ATG16L1 complex is essential for the formation of the 128 second ubiquitin-like conjugation system, where the protein light chain 3 (LC3; Atg8 in 129 yeast) is conjugated to phosphatidylethanolamine (PE) in the phagophore membrane. This 130 conjugation system relies on ATG7 (E1-like protein) and ATG3 (E2-like protein) that activate 131 LC3. In addition, ATG4B cleaves the carboxy-terminus of LC3 and exposes a glycine residue. 132 Activated LC3 is directed by ATG12-ATG5-ATG16L1 to the expanding phagophore, where 133 the E3-like activity of ATG12-ATG5 conjugates PE to the previously exposed glycine residue 134 of LC3 in the phagophore membrane. Once the phagophore is closed and the autophagosome 135 is formed, the ATG12-ATG5-ATG16L1 complex is released from the autophagosome. 136 ATG4B has also been shown to deconjugate a proportion of the LC3-PE complexes from 137 mature autophagosomes, facilitating LC3 recycling for the formation of new autophagosomes 138 (Fujita et al., 2008; Tanida et al., 2004). It is thought that recycling of LC3 is an important 139 step in the maturation of fusion-capable autophagosomes. It has also been shown that LC3 
140 mediates the hemifusion of vesicles and controls the size of autophagosomes in yeast 141 (Nakatogawa et al., 2007; Xie et al., 2008).

142 Finally, a series of fusion events of autophagosomes with endosomes and lysosomes leads 143 to their maturation into degrading autolysosomes. In mammalian cells the fusion of 144 autophagosomes with lysosomes requires the small GTPase RAB7 (Ypt7 in yeast), the 145 autophagosomal SNARE protein syntaxin 17, the lysosomal SNARE protein VAMP8 146 (vesicle-associated membrane protein 8) and several lysosomal membrane proteins, such as 147 the lysosomal-associated membrane glycoprotein 2 (LAMP2). After autophagosome148 lysosome fusion the cargo is degraded by the lysosomal hydrolases now present in the 149 autolysosome, (Eskelinen et al., 2002; Itakura et al., 2012; Jager et al., 2004; Tanaka et al., 150 2000).

151 For selective autophagy, such as xenophagy, an additional step of cargo selection is 152 required, a process that is mediated by cargo receptors and adaptor proteins. Mammalian 153 cargo specific receptors usually contain a LC3-interacting region (LIR) motif allowing to 154 recruit LC3-containing autophagosomes to the cargo (Liu et al., 2012; Novak et al., 2009; 155 Polson et al., 2014). In addition, many cargos are ubiquitinated and then they are recognized 156 by ubiquitin-binding protein adaptors that also contain LIRs. One of those proteins is p62 that, 157 among other functions, directs bacteria to autophagosomes. Together with the adaptor 158 proteins NDP52 and optineurin (OPTN), p62 is involved in S. Typhimurium recognition and 159 its direction to autophagosomes (Boyle and Randow, 2013). NDP52 and p62 also target 160 Shigella flexneri and Listeria monocytogenes to autophagosomes. Whereas p62 and NDP52 161 are recruited together during S. flexneri infection, p62 and NDP52 are recruited independently 162 during L. monocytogenes infection, suggesting that Shigella and Listeria induce different 163 pathways for selective autophagy (Mostowy et al., 2011). Another example is the neighbour 164 of BRCA1 gene 1 protein (NBR1) that is known to target Francisella tularensis to autophagy 165 (Chong et al., 2014) and also participates in the targeting of S. flexneri (Mostowy et al., 2011), 166 but it is not required for targeting of $S$. Typhimurium to autophagy (Zheng et al., 2009). These 167 studies highlight that selective autophagy uses distinct protein adaptors to target different 168 types of bacteria.

\section{Crosstalk between autophagy and the innate immune response}

170 The first step of innate immune responses is the recognition of a pathogen that is mediated by 171 a variety of pattern-recognition receptors (PRRs) like Toll-like Receptors (TLRs), RIG-I-like 172 receptors (RLRs) and NOD-like receptors (NLRs) that bind pathogen-associated molecular 
173 patterns (PAMPs) such as bacterial lipopolysaccharide (LPS), peptidoglycan, lipoproteins, 174 flagellin or nucleid acids. As autophagic degradation of pathogens aids PAMP recognition by 175 PRRs, autophagy is believed to have an important role in innate immunity. Additionally, 176 binding of PAMPs to PRRs stimulates autophagy (Delgado et al., 2009).

177 Toll-like Receptors (TLRs): TLRs are membrane-bound receptors found at the surface of 178 the cell or in endosomal compartments. TLRs respond to LPS, lipotechoic acid, flagellin and 179 bacterial nucleic acids (Deretic, 2012). After binding their cognate ligand, TLRs activate 180 proinflammatory responses by triggering the production of cytokines such as tumor necrosis 181 factor- $\alpha$ (TNF- $\alpha$ ), Interleukin-6 (IL-6) and IL-1 $\beta$ (Figure 2).

Phagocytosed and cytosolic PAMPs are sequestered by autophagosomes and are delivered to the endosomally located and luminally oriented TLRs for PAMP recognition. Therefore autophagy takes part in the first steps of TLR activation (Desai et al., 2015). On the other side, autophagy can also be induced upon TLR activation, through a MyD88/TRIF adaptor dependent process and their interaction with BECN1 (Shi et al., 2008). TLR-autophagy crosstalk is supported by several other findings: TLR4 stimulates PI3KC3-dependent formation of cytosolic LC3 aggregates, enhancing the elimination of mycobacteria from macrophages (Xu et al., 2007). Downstream of TLR4 activation, the TANK binding kinase 1 (TBK1) also links the TLR signalling pathway and autophagy as TBK1 phosphorylates the autophagic receptor OPTN, enhancing LC3 binding affinity and consequently the autophagic clearance of cytosolic Salmonella enterica (Wild et al., 2011). TLR7 activation has also been shown to promote autophagic degradation of Mycobacterium bovis BCG (Bacillus Calmette194 Guerin) (Delgado et al., 2008), and TLR2 induces autophagy in an ERK-dependent mechanism during Listeria monocytogenes infection (Anand et al., 2011). Altogether these studies highlight that TLR activation elicits autophagy, thus activating cell-autonomous immunity after PAMP detection.

However, autophagy may also down-regulate TLR-induced responses. Inhibition of autophagy in macrophages and dendritic cells (DCs) through 3MA (3-methyladenine) or through siRNA knock-down of autophagy genes, leads to an increase in cytokine release in response to TLR3 or TLR4 agonists (Harris, 2011; Saitoh et al., 2008). ATG16L1-deficient 202 mice were shown to excessively activate caspase-1 in response to TLR4 stimulation by LPS, 203 which led to increased IL-1 $\beta$ and IL-18 production. This suggests that ATG16L1 might have 204 a regulatory function on the TLR4 signalling pathway and its depletion/malfunction may lead 205 to increased inflammation (Saitoh et al., 2008). Mature IL-1 $\beta$ protein can also be engulfed by 
autophagosomes in macrophages stimulated with TLR ligands, showing another way by

207 which autophagy can downregulate the production and secretion of cytokines (Harris et al., 2011) (Figure 2). Thus, autophagy influences TLR signalling and consequently impacts TLRmediated cytokine production and secretion.

RIG-I-like receptors (RLRs): RLRs sense cytosolic dsRNA or DNA, and thus recognize

211 also nucleic acids from pathogens. PAMP recognition by RLRs as RIG-I and MDA5 triggers

212 the production of type I interferon (IFN) by infected cells, as has been shown for cells

213 infected with the intracellular pathogen Legionella pneumophila (Monroe et al., 2009). This

214 type I IFN activation pathway can be directly suppressed by several autophagy factors. For example, autophagy-defective $\operatorname{Atg} 5^{-/}$cells exhibit enhanced RLR signalling and increased IFN secretion, mostly due to the accumulation of dysfunctional mitochondria and increased generation of reactive oxygen species (ROS) which were largely responsible for the enhanced RLR signalling in $\operatorname{Atg}^{-/-}$cells (Tal et al., 2009).

cGAMP synthase (cGAS): Bacterial or aberrant cytosolic DNA are also recognized by direct binding to cGAS, a cytosolic protein that generates cyclic dinucleotides (CDNs) such as cGAMP within the host cytosol (Tao et al., 2016). Host cell-generated CDNs activate the downstream stimulator of interferon genes (STING), a receptor that can be also activated by CDNs of intracellular bacteria (Burdette et al., 2011). STING controls the activation of abovementioned TBK1, which upon nucleic acid sensing and cGAMP synthesis triggers both IFN regulatory factor 3 (IRF3) phosporylation and type I IFN production. STING induced type I IFN production occurs during infection by intracellular bacteria such as Mycobacterium tuberculosis (Watson et al., 2015). On the other hand, the autophagy activator protein ULK1 phosphorylates STING, inhibiting sustained type I IFN activation in response to dsDNA (Konno et al., 2013) (Figure 2). Furthermore, cGAS binding to BECN1 facilitates autophagic removal of cytosolic dsDNA and reduces excessive type I IFN responses (Liang et al., 2014).

NOD-like receptors (NLRS): NLRs are a class of receptors initiating a quick and potent inflammatory cytokine response to PAMPs. Upon sensing PAMPs, cytosolic NLR-receptors form a signalling complex called the inflammasome. The inflammasome consists of several oligomerized NLRs that bind Caspase-1 directly or through the adaptor protein called apoptosis-associated speck-like protein (ASC), which contains a caspase recruitment domain

236 (CARD). These complexes cleave the protein precursor pro-Caspase-1 into p10 and p20 237 subunits, activating Caspase-1. Active Caspase-1 then cleaves the presynthesized pro-IL-1 $\beta$ 238 into the active form of the cytokine IL-1 $\beta$, which is secreted (Rodgers et al., 2014). IL-1 $\beta$ 
secretion relies on two different PRR activating signals: the NF- $\kappa \mathrm{B}$-dependant expression of pro-IL-1 $\beta$ through TLR activation and a signal activating Caspase-1 through inflammasomes

241 to cleave pro-IL-1 $\beta$ (Eder, 2009) (Figure 2). The inflammasome also cleaves pro-IL-18 into 242 active IL-18 and orchestrates the programmed cell death known as pyroptosis (Miao et al., 243 2011). Therefore, the inflammasome promotes inflammation by controlling the secretion of 244 the strong proinflammatory cytokines IL-1 $\beta$ and IL-18 and avoiding that cells become a niche 245 for the pathogens by activating pyroptotic cell death. These two cytokines are responsible for 246 the recruitment of myeloid cells, including neutrophils, to sites of inflammation (Rider et al., 247 2011).

248 NLRs that respond to PAMPs in macrophages and activate Caspase-1 by inflammasome 249 assembly are NLRP1, NLRP3, NLRC4, NLRP7, NLRP12 and AIM2 (absent in melanoma 2) 250 (Latz et al., 2013). Interestingly, a vast amount of data strongly suggests that both machineries, 251 autophagy and inflammasome, are highly interconnected and influence each other. Remnants 252 of autophagosomes that were degraded during S. flexneri infection of epithelial cells are 253 decorated with components of both, the autophagy and the inflammasome machineries. 254 Moreover, macrophages of Caspase- $1^{-/}$mice display an excessive accumulation of 255 autophagosomes during $S$. flexneri infection, suggesting that Caspase-1 affects either 256 autophagosome formation or maturation (Dupont et al., 2009; Suzuki et al., 2007). Activation 257 of AIM2 with poly(dA:dT) or NLRP3 activation with uric acid crystals or nigericin leads to 258 an increase in autophagosome formation in macrophages (Shi et al., 2012). Autophagosomes 259 have also been shown to sequester and degrade inflammasome components and cytokine 260 precursors such as pro-IL-1 $\beta$ (Harris et al., 2011; Shi et al., 2012). Association between 261 autophagy and inflammasome proteins was also shown in resting cells, where NLRP4 and 262 NLRC4 formed a complex with BECN1, suggesting that NLRP4 sensing of bacteria leads to 263 the initiation of BECN1-mediated autophagic responses (Jounai et al., 2011). Autophagy and 264 inflammation are also regulated by NOD1 and NOD2, a Caspase-1 independent class of NLR 265 proteins. NOD1 and NOD2 are intracellular sensors of peptidoglycan that induce autophagy

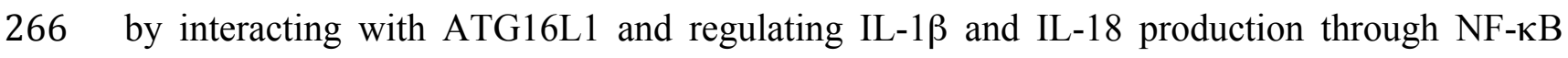
267 (Philpott et al., 2013).

268 Key evidence for NLR signalling in anti-bacterial responses was gained from the analysis of 269 mouse susceptibility to L. pneumophila. Indeed, only A/J mice are susceptible to infection 270 due to a partial loss-of-function mutation in the Naip5 inflammasome, a component of the 
271 flagellin-NLCR4 pathway (Molofsky et al., 2006; Zamboni et al., 2006). Autophagosomes of

272 macrophages from L. pneumophila-resistant C57BL/6J mice matured quickly and prevented

273 efficient L. pneumophila replication when compared to autophagosomes of L. pneumophila-

274 permissive Naip5 mutant A/J macrophages. This observation is reinforcing the idea that

275 inflammasome activation and autophagy are intertwined processes during bacterial infection

276 (Amer and Swanson, 2005). Additional results obtained in primary mouse macrophages

277 support a model in which both Caspase-1 and NLR components of inflammasomes are co-

278 ordinately responding to L. pneumophila infection depending on the bacterial burden (Byrne

279 et al., 2013). According to this model, NLRC4 is complexed with autophagy component

280 BECN1 in resting macrophages, inhibiting autophagy. Low levels of bacteria lead flagellin-

281 bound NAIP5 to recruit NLRC4 to a complex containing the pro-Caspase-1 protein,

282 derepressing autophagy. When the capacity of autophagy to eliminate intracellular bacteria is

283 exceeded, Caspase-1 triggers pyroptosis to eliminate the pathogen's niche while initiating a

284 potent inflammatory response (Byrne et al., 2013).

\section{Autophagy crosstalks with adaptive immune responses}

286 Considering the role of autophagy in the restriction and destruction of intracellular pathogens and its interplay with innate immunity, it is expected that autophagy is also a crucial process in adaptive immunity where it acts on the modulation of antigen processing and presentation to elicit the correct development and homeostasis of lymphocytes.

$\mathrm{T}$ cells are the main effectors of the adaptive immune system. They scan through the output of the proteolytic machineries of the cells to detect pathogen-derived peptides. CD4 ${ }^{+}$

292 helper $\mathrm{T}$ cells and cytotoxic $\mathrm{CD}^{+} \mathrm{T}$ cells display a diverse receptor repertoire that allows 293 them to recognize these peptides but this recognition is not direct as antigens need to be 294 loaded to major histocompatibility class (MHC) molecules before being presented to T cells. 295 MHC class I ligands are commonly generated by the proteasome and are presented to $\mathrm{CD}^{+} \mathrm{T}$ 296 cells while MHC class II loading peptides are produced by lysosomes and presented to CD4 ${ }^{+}$ 297 T cells (Münz, 2010).

298 The classical concept suggests that intracellular antigens get processed and loaded onto 299 MHC class I cells while extracellular ones go on MHC class II cells. However, as autophagy delivers cytoplasmic constituents for lysosomal degradation and MHC class II molecules are loaded with lysosomal products, autophagy also supports the processing of endogenous antigens for presentation by MHC class II (Dengjel et al., 2005; Nimmerjahn et al., 2003; 
303 Schmid et al., 2007; Zhou et al., 2005). Therefore, antigen-presenting cells such as 304 macrophages and DCs can use the autophagy machinery to fuse autophagosomes containing bacterial-derived antigens with autolysosomes, which are afterwards loaded into MHC class II molecules for antigen presentation to $\mathrm{CD}^{+} \mathrm{T}$ cells (Crotzer and Blum, 2009; Dengjel et al., 2005). This antigen-presentation enhancing effect of autophagy has been used to significantly upgrade the efficiency of the BCG vaccine (Jagannath et al., 2009). Autophagy also regulates exogenous antigen processing for presentation by MHC class II via the modification of the content and fate of phagosomes after LC3 recruitment to the phagosomal membranes 311 (Shibutani et al., 2015).

312 T cells may upregulate autophagy upon T-cell receptor (TCR) stimulation, a process that 313 seems to be essential not only for T cell proliferation but also for their survival as autophagy314 defective $\mathrm{CD}^{+}$cells are more susceptible to apoptosis (Kovacs et al., 2011; Pua et al., 2007). 315 One important physiological process in which autophagy-dependent endogenous antigen 316 presentation by MHC class II is essential is the education of naïve CD4+ T cells in the thymus, 317 where thymic epithelial cells (TECs) present self-antigens on MHC molecules for the 318 induction of T cell tolerance. TECs display constitutive starvation-independent high levels of autophagy which appear to be crucial for correct negative selection of $\mathrm{T}$ cells, elimination of autoreactive $\mathrm{T}$ cells and the correct development of self-tolerance (Aichinger et al., 2013; 321 Nedjic et al., 2008).

However, autophagy pathways engage in far more aspects of adaptive immunity than antigen presentation, as they also affect lymphocyte selection, maturation, proliferation and survival. Defects in autophagy lead to serious damage in the lymphoid lineage: mice lacking ATG5 displayed a reduced number of B and T cells, suggesting that autophagy regulates the activity of lymphoid precursors. Even though lethally irradiated mice get repopulated with haematopoietic cells of $\mathrm{Atg}^{-/-}$mice, the $\mathrm{CD}^{+}$and $\mathrm{CD}^{+}$cells failed to undergo proliferation upon T-receptor stimulation. $\operatorname{Atg} 5^{-/} \mathrm{T}$ cells managed to repopulate the thymus but experienced high levels of cell death that prevented the repopulation of the periphery (Pua et al., 2007). ATG7-deficient cells failed to reconstitute the haematopoietic system of lethally irradiated mice and the production of lymphoid progenitors was also impaired in the absence of ATG7 (Mortensen et al., 2011). Selective autophagy of mitochondria (mitophagy), also seems to be essential for the development of $T$ cells as the number of naïve $T$ cells is 334 significantly lower if mitophagy is impaired (Farfariello et al., 2012; Pua et al., 2009). 335 Furthermore, the absence of autophagy during B cell differentiation appears to negatively 
336 impact the numbers of B1 cells while the overall number of B cells remains unchanged, a 337 process that seems to involve BECN1 and ATG5 (Arsov et al., 2011; Miller et al., 2014). 338 Finally, autophagy is capital for the maintenance of plasma cells, which require autophagy for 339 sustainable immunoglobulin production (Pengo et al., 2013).

\section{Autophagy regulates cytokine production during bacterial infection}

341 Considering the crosstalk between autophagy and PRR signalling, it can be hypothesized that 342 autophagy influences cytokine production during bacterial infection. Indeed, several groups 343 investigated the effect of autophagy inhibition on cytokine production, either during in vitro 344 infection of isolated cells or in vivo infection of mice with bacterial pathogens (Table 1).

345 These studies have shown that inhibition of autophagy by 3MA decreased TNF- $\alpha$ production 346 but enhanced IL-1 $\beta$ and IL-6 production in $M$. tuberculosis-infected peripheral blood 347 mononuclear cells (PBMCs). In contrast, induction of autophagy by starvation had the 348 opposite effect (Kleinnijenhuis et al., 2011). Similar results were obtained for 3MA inhibition 349 of autophagy during infection of PBMCs with Borrelia burgdorferi, the causative agent of 350 Lyme disease, as IL-1 $\beta$ and IL-6 increased while TNF- $\alpha$ remained unaltered (Buffen et al., 351 2013). In a mouse model of Pseudomonas aeruginosa infection, Li and colleagues showed 352 that Annexin A2 (AnxA2) regulates autophagosome formation through the mTORC1-ULK1 353 signalling pathway (Li et al., 2015) as infected Anxa2 $2^{-/-}$mice displayed reduced autophagy 354 levels and a marked increase of cytokines, in particular IL-1 $\beta$, TNF- $\alpha$, IL- 6 and IFN- $\gamma$, in 355 bronchoalveolar lavage fluid (BALF). Given the body of work available, it seems plausible to 356 affirm that autophagy reduction induces an increase in cytokine production during bacterial 357 infection.

358 Chron's disease is an inflammatory disorder characterized by an excessive immune 359 response to intestinal microbiota. The Thr300Ala polymorphism on ATG16L1 is associated 360 with Chron's disease, a fact that inspired several studies on cytokines production on 361 ATG16L1 downregulation conditons (Conway et al., 2013; Lapaquette et al., 2012; Lassen et 362 al., 2014). One of the first studies showed that knockdown of Atg16l1 in THP-1 macrophage363 like cells led to decreased autophagy and increased production of TNF- $\alpha$ and IL-6 during 364 infection with adherent invasive Escherichia coli (Lapaquette et al., 2012). In a later study, 365 Conway and colleagues showed that S.Typhimurium was unable to associate with 366 autophagosomes in Atg16l1-deficient epithelial cells and showed significantly higher levels of 367 IL-1 $\beta$ and IL-6 compared to wild-type (WT) mice in the terminal ileum and cecum (Conway 368 et al., 2013). But the most informative study was carried out using a knock-in mouse model 
expressing the ATG16L1 T300A variant (Lassen et al., 2014). As autophagy was reduced in multiple cell types from T300A knock-in mice compared to WT mice, the authors explored several aspects of the immune response to $S$. flexneri infection. Their results showed that the T300A polymorphism was associated with decreased antibacterial autophagy and increased IL-1 $\beta$ production in primary cells and in vivo. Interestingly, in vitro bacterial infections of

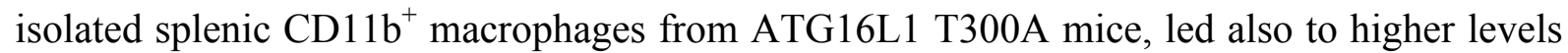
of IL-1 $\beta$. Collectively, this study shows that defective autophagy caused by a diseaseassociated polymorphism of an autophagy gene leads to increased IL-1 $\beta$ production. However, the mechanism linking ATG16L1 to IL-1 $\beta$ secretion was not elucidated (Lassen et al., 2014).

Furthermore, a recent report showed that injection of $B$. burgdorferi into the knees of $\operatorname{Atg} 7^{-/-}$mice increased joint swelling and cytokine levels (IL-1 $\beta$ and IL-6) when compared to WT mice, suggesting that B. burgdorferi-induced joint inflammation is controlled by autophagy (Buffen et al., 2016). Autophagy inhibition by wortmannin led to an increase in the production of IL-1 $\beta$ and IL-23 cytokines by human PBMCs infected with $B$. burgdorferi suggesting that the production of these cytokines is controlled by autophagy during B. burgdorferi infection in vitro. Increased production of both cytokines, IL-1 $\beta$ and IL-23, led to the polarization of $\mathrm{CD}^{+} \mathrm{T}$ cells to IL-17-producing Th17 cells, a specific subtype of T cells that is commonly elevated in patients with confirmed neuroborreliosis (Henningsson et al., 2011) and which seem to be involved in the pathogenesis of Lyme arthritis (Burchill et al., 2003). Collectively, these results show that autophagy controls B. burgdorferi-induced secretion of cytokines such as IL-1 $\beta$ and IL-23, which in turn impact T-cell polarization during infection.

Thus, it seems that autophagy has a role limiting cytokine production during bacterial infection (especially IL-1 $\beta$ production). This resembles the cytokine production profiles observed during stimulation of cells with bacterial ligands in the presence of autophagy inhibitors discussed in previous sections.

\section{Intracellular bacteria modulate autophagy in the infected cell}

Infection by bacterial pathogens triggers autophagy in infected cells as a cell-autonomous defence mechanism aimed at degrading the invading pathogen. However, pathogenic bacteria evolved mechanisms to manipulate autophagy and counteract these host self-defences. While certain intracellular bacteria induce and manipulate autophagy taking advantage of it, other 
al., 2016).

Anaplasma phagocytophilum, Yersinia pseudotuberculosis, Coxiella burnetii and

403 F.tularensis are intracellular pathogens that have evolved mechanisms to hijack 404 autophagosomes during infection. These bacteria redirect the by-products of the autophagic degradation of cellular components for their own nutritional use, thereby promoting their replication (Steele et al., 2015). They replicate within bacterial vacuoles decorated with autophagy components, such as LC3, and show defective replication in autophagy-deficient cells. Consequently, treatment of host cells with autophagy activators, increases bacterial replication rather than promoting bacterial clearance (Escoll et al., 2016). A. phagocytophilum uses the secreted effector Ats-1 to promote autophagosome nucleation and utilization of the nutrients contained in the autophagosomes (Niu et al., 2012). Similarly, it was elegantly shown by monitoring autophagy-derived radiolabeled amino acids that during $F$. tularensis infection a transfer from host proteins to invading bacteria takes place (Steele et al., 2013).

Other bacteria, such as $M$. tuberculosis and $S$. Typhimurium, inhibit autophagy initiation upstream of autophagosome formation, thus evading xenophagy and pathogen degradation (Shin et al., 2010; Tattoli et al., 2012). S. flexneri evades autophagy recognition by masking the bacterial surface (Ogawa et al., 2005).

An important observation is that some bacterial pathogens actively induce autophagy but, 419 at the same time, block autophagosome maturation and fusion with the lysosome. L. pneumophila is one of these intracellular bacterial pathogens that uses this dual strategy.

421 After phagocytosis, the establishment of the Legionella-containing vacuole (LCV) in the 422 infected macrophage is accompanied by the acquisition of autophagy markers like LC3 at the 423 LCV, showing that the LCVs rapidly become autophagosomes (Amer and Swanson, 2005).

424 The secreted L. pneumophila effector LegA9 was shown to promote the recognition of the 425 LCV by the autophagy machinery (Khweek et al., 2013). In line with this observation, inhibition of autophagy in permissive A/J mouse macrophages reduces L. pneumophila survival at $2 \mathrm{~h}$ post-infection (Amer and Swanson, 2005; Amer et al., 2005), supporting the idea that autophagy has a role in the promotion of the survival of L. pneumophila within the host cell early during infection (Amer et al., 2005). Later during infection, L. pneumophila inhibits autophagy by secreting the effectors LpSPL and RavZ (Choy et al., 2012; Rolando et 431 al., 2016), which respectively inhibit autophagosome formation and maturation. This strategy delays the maturation of the LCV-containing autophagosome into autolysosomes, thus gaining precious time for multiplication of the pathogen (Amer and Swanson, 2005; Joshi and 
Swanson, 2011).

\section{Autophagy subversion by pathogenic bacteria shapes host immunity}

As previously discussed, autophagy and immunity are strongly linked and coordinated processes. Considering that many pathogenic bacteria manipulate autophagy, it is expected that this strategy has direct consequences on immunity. Regarding cell-autonomous immunity, bacteria-induced inhibition of autophagy promotes evasion from xenophagy, a benefit for the pathogen as they escape lysosomal degradation. However, autophagy is also linked to "conventional" immune responses, i.e. innate and adaptive immunity (Kuballa et al., 2012) but this relationship and the impact of bacterial modulation of autophagy on immune responses remains poorly understood.

Manipulation of autophagy by pathogenic bacteria may impact cytokine production by innate immune cells, as autophagy is interconnected to PRR signalling. This may represent an underappreciated effect on innate immunity induced by bacteria that modulate autophagy. Indeed as listed in Table 1 some studies have pointed to this possibility. For example, the M. tuberculosis protein Eis is involved in bacterial survival within the host. Macrophages infected with a mutant lacking eis displayed markedly increased accumulation of autophagy vacuoles and formation of autophagosomes in vitro and in vivo, suggesting that Eis downregulates autophagy in the host cell (Shin et al., 2010). Interestingly, infection of macrophages with a $\Delta$ eis mutant strain increased the production of TNF- $\alpha$ and IL-6 as compared to those measured after infection with the WT strain, suggesting that the loss of the capacity of the $\Delta$ eis mutant to inhibit autophagy leads to the increased TNF- $\alpha$ and IL-6 production (Shin et al., 2010). In addition, the virulence factor TlyA also inhibits autophagy and significantly contributes to the pathogenesis of M. tuberculosis. DCs infected with a $\Delta$ tly $A$ strain displayed increased autophagy and showed increased IL-12p40 and reduced IL$1 \beta$ and IL-10 cytokine responses, which clearly contrasts with the immune responses induced by the WT strain (Rahman et al., 2015). Collectively, these two studies suggest that inhibition of autophagy induced by Eis and TlyA reduces production of TNF- $\alpha$, IL-6 and IL-12p40 while it boosts the secretion of IL-1 $\beta$ and IL-10 in response to M. tuberculosis infection.

The bacterial effector LpSPL secreted by L. pneumophila inhibits autophagy in human cells by modulating the sphingolipid metabolism of the host cell during infection (Rolando et al., 2016). It was also shown, that a mutant lacking this effector induces higher secretion of IL-1 $\beta$ and lower secretion of TNF- $\alpha$, IL-6 and IL-10 compared to the WT strain during 
466 infection of BMDMs (Abu Khweek et al., 2016). As these studies were carried out in 467 different host cells (human and mice) and none of them tested the influence of 468 L. pneumophila-induced inhibition of autophagy on cytokine production of infected cells 469 directly, it is difficult to draw conclusions about how manipulation of autophagy by 470 L. pneumophila influences cytokine production of infected macrophages, however, it is 471 tempting to assume that there is a link.

472 As autophagy is also involved in antigen presentation to $\mathrm{T}$ cells, autophagy modulation by 473 bacterial pathogens may also impact adaptive immunity. Indeed, a recent report showed that 474 autophagy modulation by $M$. tuberculosis impacts adaptive immunity. A genome-wide 475 screening identified the protein PE PGRS47 of $M$. tuberculosis as responsible for the inhibition of MHC class II antigen presentation of infected DCs as $\triangle P E_{-} P G R S 47$-infected

477 DCs showed increased MHC class II antigen presentation compared to the WT strain, and 478 infection of mice with the $\triangle P E_{-} P G R S 47$ strain resulted in an increased number of bacterial479 antigen-specific $\mathrm{CD}^{+}$cells compared to WT-infected mice (Saini et al., 2016). Interestingly, 480 infection with a M. tuberculosis mutant lacking PE_PGRS47 showed increased autophagy 481 when compared to the WT strain, suggesting that the bacteria use PE_PGRS47 to inhibit autophagy in the host cell. Moreover, the $\triangle P E_{-} P G R S 47$ strain was significantly attenuated in vivo and its defects in intracellular replication in vitro were restored to WT levels when autophagy was inhibited by 3MA treatment (Saini et al., 2016). These results demonstrate that 485 the M. tuberculosis protein PE_PGRS47 inhibits autophagy in the infected cells resulting in a reduction of MHC class II antigen presentation that impacts specific T-cell responses to infection.

488 Concluding remarks

489 Autophagy works as a cell-autonomous defence mechanism of immune and non-immune cells 490 by removing invading pathogens immediately after infection. It is well established that 491 bacterial-induced inhibition of autophagy promotes bacterial evasion from xenophagy and 492 allows the pathogen to escape from lysosomal degradation (Huang and Brumell, 2014). 493 Autophagy is also linked to conventional innate and adaptive immune responses but the 494 impact of autophagy modulation by bacteria at these levels remains poorly understood.

495 Current data support a model where PRR signalling and autophagy crosstalk at different levels. In general, PRR activation through the recognition of bacterial ligands promotes 497 autophagy (Delgado et al., 2009). However, drugs or genetic approaches used to inhibit 
autophagy in infected cells or animals showed increased secretion of pro-inflammatory cytokines after stimulation with bacterial ligands or viable bacteria, suggesting that cells might also use autophagy to limit PRR-initiated immune responses as a negative-feedback

501 loop during infection. Most of the available literature supports this model (Table 1), with data 502 for inflammasome signalling and IL-1 $\beta$ secretion being very solid and reproducible through 503 out the different studies.

504 One of the most compelling results is the role of ATG16L1 in Chron's disease. Defects in 505 ATG16L1, such as the T300A polymorphism carried by patients, lead to reduced autophagy 506 in host cells during bacterial infection, reduced bacterial clearance and increased secretion of 507 cytokines, mainly of IL-1 $\beta$ (Conway et al., 2013; Lapaquette et al., 2012; Lassen et al., 2014). 508 These studies highlight the existence of intrinsic mechanisms within host cells where 509 autophagy and cytokine production are coordinated during host-pathogen interactions, 510 suggesting that malfunction of autophagy might be one cause of inflammatory diseases with 511 excessive cytokine production such as Chron's disease. Finally, the investigation of the 512 mechanisms used by bacteria to modulate autophagy identified some bacterial effectors 513 inhibiting autophagy, thereby promoting bacterial survival during infection. However, only 514 few studies addressed the question of how these bacterial-derived autophagy inhibitors 515 directly impact immune responses during infection. Most is known for M. tuberculosis, in 516 which the bacterial proteins Eis, TlyA and PE_PGRS47 have been shown to inhibit 517 autophagy and to modulate cytokine production and MHC class II antigen presentation to T 518 cells, shaping innate and adaptive immunity during $M$. tuberculosis infection.

519 Future work on this topic should be directed to uncover whether mechanisms exist that coordinate autophagy and cytokine production during infection. It would be important to 521 investigate in parallel the impact of autophagy on the secretion of inflammasome-dependent/522 independent cytokines (such as IL-1 $\beta$ and TNF- $\alpha$, respectively) and also on the production of 523 anti-inflammatory cytokines such as IL-10 or TGF- $\beta$. Additionally, an intriguing question yet 524 to be answered is whether or not bacterial effectors that increase or reduce autophagy (such 525 Ats-1 or $L p$ SPL, respectively) impact the innate and adaptive immune responses to infection. 526 In depth knowledge on these questions will help to better understand bacterial infection and to 527 better combat disease.

\section{Acknowledgements}

530 Work in CB laboratory is financed by the Institut Pasteur, the Institut Carnot-Pasteur MI, the 
531 French Region Ile de France (DIM Malinf) and the grant $n^{\circ}$ ANR-10-LABX-62-IBEID. FV 532 was funded by the Infect-ERA project EUGENPATH (ANR-13-IFEC-0003-02), PE by the 533 Fondation pour la Recherche Médicale, grant N DEQ20120323697.

\section{References}

Abu Khweek, A., Kanneganti, A., Guttridge D, D.C., and Amer, A.O. (2016). The Sphingosine-1-Phosphate Lyase (LegS2) Contributes to the Restriction of Legionella pneumophila in Murine Macrophages. PLoS ONE 11, $538 \mathrm{e} 0146410$

Aichinger, M., Wu, C., Nedjic, J., and Klein, L. (2013). Macroautophagy substrates are loaded onto MHC class II of medullary thymic epithelial cells for central tolerance. Journal of Experimental Medicine 210, 287-300.

Amer, A.O., and Swanson, M.S. (2005). Autophagy is an immediate macrophage response to Legionella pneumophila. Cellular Microbiology 7, 765-778.

Amer, A.O., Byrne, B.G., and Swanson, M.S. (2005). Macrophages rapidly transfer pathogens from lipid raft

Arsov, I., Arsov, I., Adebayo, A., Adebayo, A., Kucerova-Levisohn, M., Kucerova-Levisohn, M., Haye, J., Haye, J., MacNeil, M., MacNeil, M., et al. (2011). A Role for Autophagic Protein Beclin 1 Early in Lymphocyte Development. J. Immunol. 186, 2201-2209.

548 Buffen, K., Oosting, M., Li, Y., Kanneganti, T.-D., Netea, M.G., and Joosten, L.A.B. (2016). Autophagy 549 suppresses host adaptive immune responses toward Borrelia burgdorferi. J. Leukoc. Biol. 100, 589-598.

Byrne, B.G., Dubuisson, J.-F., Joshi, A.D., Persson, J.J., and Swanson, M.S. (2013). Inflammasome components coordinate autophagy and pyroptosis as macrophage responses to infection. mBio 4, e00620-12.

Choy, A., Dancourt, J., Mugo, B., O'Connor, T.J., Isberg, R.R., Melia, T.J., and Roy, C.R. (2012). The 1076.

Crotzer, V.L., and Blum, J.S. (2009). Autophagy and its role in MHC-mediated antigen presentation. The

556 Journal of Immunology 182, 3335-3341.

Dengjel, J., Dengjel, J., Schoor, O., Schoor, O., Fischer, R., Fischer, R., Reich, M., Reich, M., Kraus, M., Kraus, M., et al. (2005). Autophagy promotes MHC class II presentation of peptides from intracellular source proteins.

559 Proc. Natl. Acad. Sci. U.S.a. 102, 7922-7927.

560 Deretic, V., Saitoh, T., and Akira, S. (2013). Autophagy in infection, inflammation and immunity. Nat. Rev. 561 Immunol. 13, 722-737.

562 Desai, M., Fang, R., and Sun, J. (2015). The role of autophagy in microbial infection and immunity.

563 Immunotargets Ther 4, 13-26.

564 Escoll, P., Rolando, M., and Buchrieser, C. (2016). Modulation of Host Autophagy during Bacterial Infection:

565 Sabotaging Host Munitions for Pathogen Nutrition. Front Immunol 7, 81.

566 Farfariello, V., Farfariello, V., Amantini, C., Amantini, C., Santoni, G., and Santoni, G. (2012). Transient 567 receptor potential vanilloid 1 activation induces autophagy in thymocytes through ROS-regulated AMPK and 568 Atg4C pathways. Journal of Leukocyte Biology 92, 421-431.

569 Harris, J., Hartman, M., Roche, C., Zeng, S.G., O'Shea, A., Sharp, F.A., Lambe, E.M., Creagh, E.M., Golenbock, 570 D.T., Tschopp, J., et al. (2011). Autophagy controls IL-1beta secretion by targeting pro-IL-1beta for degradation. 571 Journal of Biological Chemistry 286, 9587-9597. 

114.

Jagannath, C., Lindsey, D.R., Dhandayuthapani, S., Xu, Y., Hunter, R.L., and Eissa, N.T. (2009). Autophagy enhances the efficacy of BCG vaccine by increasing peptide presentation in mouse dendritic cells. Nat Med 15, $267-276$.

Joshi, A.D., and Swanson, M.S. (2011). Secrets of a successful pathogen: legionella resistance to progression along the autophagic pathway. Front. Microbio. 2, 138.

Khweek, A.A., Caution, K., Akhter, A., Abdulrahman, B.A., Tazi, M., Hassan, H., Majumdar, N., Doran, A.,

580 Guirado, E., Schlesinger, L.S., et al. (2013). A bacterial protein promotes the recognition of the Legionella

581 pneumophila vacuole by autophagy. Eur. J. Immunol. 43, 1333-1344.

Kovacs, J.R., Li, C., Yang, Q., Li, G., Garcia, I.G., Ju, S., Roodman, D.G., Windle, J.J., Zhang, X., and Lu, B. (2011). Autophagy promotes T-cell survival through degradation of proteins of the cell death machinery. Cell Death Differ. 19, 144-152.

Kuballa, P., Nolte, W.M., Castoreno, A.B., and Xavier, R.J. (2012). Autophagy and the Immune System. Annu. Rev. Immunol. 30, 611-646.

Latz, E., Xiao, T.S., and Stutz, A. (2013). Activation and regulation of the inflammasomes. Nat. Rev. Immunol. $13,397-411$.

Miller, B.C., Zhao, Z., Stephenson, L.M., Cadwell, K., Pua, H.H., Lee, H.K., Mizushima, N., Iwasaki, A., He, Y.-W., Swat, W., et al. (2014). The autophagy gene ATG5plays an essential role in B lymphocyte development. 591 Autophagy 4, 309-314.

Miller, C., and Celli, J. (2016). Avoidance and Subversion of Eukaryotic Homeostatic Autophagy Mechanisms by Bacterial Pathogens. J. Mol. Biol. 428, 3387-3398.

Molofsky, A.B., Byrne, B.G., Whitfield, N.N., Madigan, C.A., Fuse, E.T., Tateda, K., and Swanson, M.S. (2006). Cytosolic recognition of flagellin by mouse macrophages restricts Legionella pneumophila infection. Journal of Experimental Medicine 203, 1093-1104.

Monroe, K.M., McWhirter, S.M., and Vance, R.E. (2009). Identification of Host Cytosolic Sensors and Bacterial 598 Factors Regulating the Type I Interferon Response to Legionella pneumophila. PLoS Pathog 5, e1000665.

599 Mortensen, M., Soilleux, E.J., Djordjevic, G., Tripp, R., Lutteropp, M., Sadighi-Akha, E., Stranks, A.J., 600 Glanville, J., Knight, S., W Jacobsen, S.-E., et al. (2011). The autophagy protein Atg7 is essential for 601 hematopoietic stem cell maintenance. Journal of Experimental Medicine 208, 455-467.

602 Münz, C. (2010). Antigen processing via autophagy—not only for MHC class II presentation anymore? Current 603 Opinion in Immunology 22, 89-93.

604 Nedjic, J., Aichinger, M., Emmerich, J., Mizushima, N., and Klein, L. (2008). Autophagy in thymic epithelium 605 shapes the T-cell repertoire and is essential for tolerance. Nature 455, 396-400.

606 Nimmerjahn, F., Milosevic, S., Behrends, U., Jaffee, E.M., Pardoll, D.M., Bornkamm, G.W., and Mautner, J. 607 (2003). Major histocompatibility complex class II-restricted presentation of a cytosolic antigen by autophagy. 608 Eur. J. Immunol. 33, 1250-1259.

609 Ohsumi, Y. (2014). Historical landmarks of autophagy research. Cell Res 24, 9-23.

610 Pengo, N., Scolari, M., Oliva, L., Milan, E., Mainoldi, F., Raimondi, A., Fagioli, C., Merlini, A., Mariani, E., 611 Pasqualetto, E., et al. (2013). Plasma cells require autophagy for sustainable immunoglobulin production. Nat 612 Immunol 14, 298-305.

613 Pua, H.H., Pua, H.H., Guo, J., Guo, J., Komatsu, M., Komatsu, M., He, Y.-W., and He, Y.W. (2009). Autophagy 614 Is Essential for Mitochondrial Clearance in Mature T Lymphocytes. J. Immunol. 182, 4046-4055. 
615 Pua, H.H., Dzhagalov, I., Chuck, M., Mizushima, N., and He, Y.-W. (2007). A critical role for the autophagy

616 gene Atg5 in T cell survival and proliferation. Journal of Experimental Medicine 204, 25-31.

617 Rolando, M., Escoll, P., Nora, T., Botti, J., Boitez, V., Bedia, C., Daniels, C., Abraham, G., Stogios, P.J.,

618 Skarina, T., et al. (2016). Legionella pneumophila S1P-lyase targets host sphingolipid metabolism and restrains

619 autophagy. Proc. Natl. Acad. Sci. U.S.a. 113, 1901-1906.

620 Rubinsztein, D.C., Codogno, P., and Levine, B. (2012). Autophagy modulation as a potential therapeutic target

621 for diverse diseases. Nat Rev Drug Discov 11, 709-730.

622 Saini, N.K., Baena, A., Ng, T.W., Venkataswamy, M.M., Kennedy, S.C., Kunnath-Velayudhan, S., Carreño, L.J.,

623 Xu, J., Chan, J., Larsen, M.H., et al. (2016). Suppression of autophagy and antigen presentation by

624 Mycobacterium tuberculosis PE_PGRS47. Nature Microbiology 1, 16133.

625 Schmid, D., Pypaert, M., and Münz, C. (2007). Antigen-Loading Compartments for Major Histocompatibility

626 Complex Class II Molecules Continuously Receive Input from Autophagosomes. Immunity 26, 79-92.

627 Shi, C.-S., Shenderov, K., Huang, N.-N., Kabat, J., Abu-Asab, M., Fitzgerald, K.A., Sher, A., and Kehrl, J.H.

628 (2012). Activation of autophagy by inflammatory signals limits IL-1 $\beta$ production by targeting ubiquitinated

629 inflammasomes for destruction. Nat Immunol 13, 255-263.

630 Shibutani, S.T., Saitoh, T., Nowag, H., Münz, C., and Yoshimori, T. (2015). Autophagy and autophagy-related 631 proteins in the immune system. Nat Immunol 16, 1014-1024.

632 Zamboni, D.S., Kobayashi, K.S., Kohlsdorf, T., Ogura, Y., Long, E.M., Vance, R.E., Kuida, K., Mariathasan, S., 633 Dixit, V.M., Flavell, R.A., et al. (2006). The Bircle cytosolic pattern-recognition receptor contributes to the 634 detection and control of Legionella pneumophila infection. Nat Immunol 7, 318-325.

635 Zhou, D., Li, P., Lin, Y., Lott, J.M., Hislop, A.D., Canaday, D.H., Brutkiewicz, R.R., and Blum, J.S. (2005).

636 Lamp-2a Facilitates MHC Class II Presentation of Cytoplasmic Antigens. Immunity 22, 571-581. 
639 Figure 1. Schematic description of the autophagic pathway. Autophagy is triggered by 640 starvation, damaged cellular components or cellular recognition of bacteria. These events 641 activate the ULK1 complex. Under nutrient-rich conditions, mTORC1 inhibits 642 autophagosome formation by repressing the ULK1 complex, however participation of 643 mTORC1 in bacterial-induced autophagy is unclear. Once the ULK1 complex is activated, 644 autophagosome formation begins with the recruitment of BECN1 and the nucleation of a 645 small membrane called the phagophore, which starts to engulf the undesired material. The 646 phagophore expands to form the autophagosome, a double-membrane compartment engulfing 647 cyoplasmic targets (proteins, organelles or pathogens). This mature autophagosome then fuses 648 with a lysosome for cargo degradation.

649 Figure 2. Crosstalk of autophagy and innate immunity. Activation of PRRs, such as TLRs, 650 inflammasomes and cGAS, triggers signalling pathways (green components) that lead to 651 activation of inflammatory transcription factors (orange components) and autophagy proteins 652 that activate autophagy (brown components). Activation of inflammatory transcription factors 653 leads to transcription and translation of cytokines such as TNF- $\alpha$, IL-6 and IFN- $\gamma$, which are 654 secreted, but also pro-IL-1 $\beta$ and pro-IL-18, which are then processed by inflammasome655 activated Caspase-1 into secreted IL-1 $\beta$ and IL-18. In turn, autophagy aids TLRs in meeting 656 their cognate ligands, while it negatively regulates cytokine production by repressing cGAS 657 synthesis of cGAMP and degrading precursor and mature forms of IL-1 $\beta$ and IL-18, and 658 inflammasome components (dashed lines) 


\begin{tabular}{|c|c|c|c|c|c|c|c|c|c|c|c|c|}
\hline 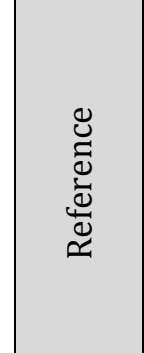 & 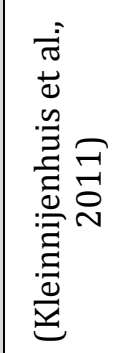 & \multicolumn{2}{|c|}{ 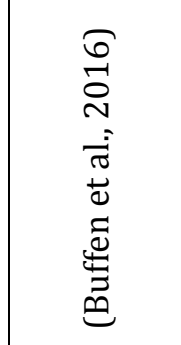 } & 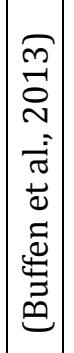 & 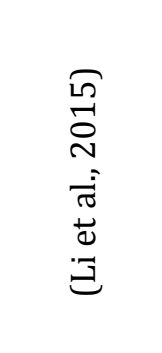 & 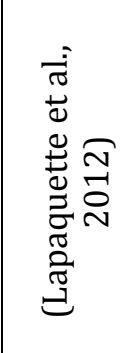 & 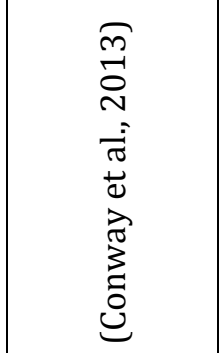 & 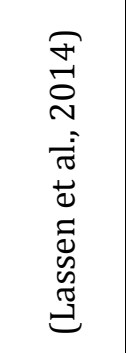 & 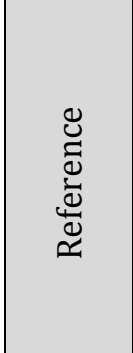 & 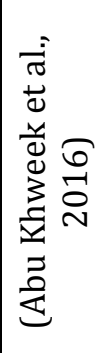 & 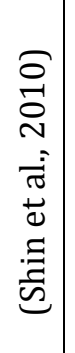 & 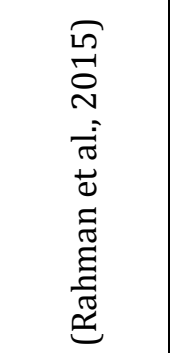 \\
\hline 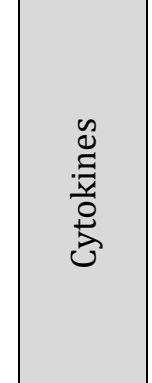 & 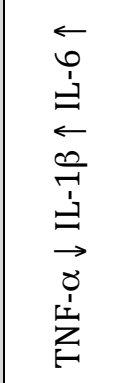 & 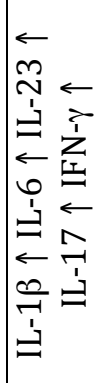 & 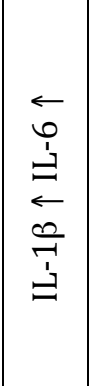 & 玄 & 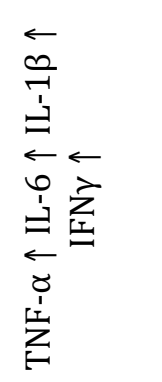 & 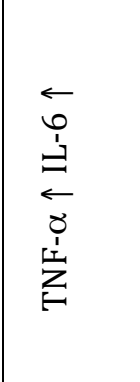 & 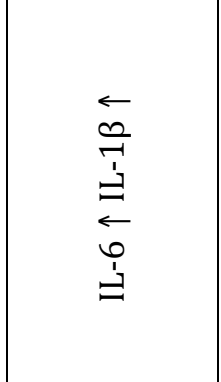 & $\begin{array}{l}\stackrel{\leftarrow}{\leftrightarrows} \\
\stackrel{\leftrightarrow}{=}\end{array}$ & 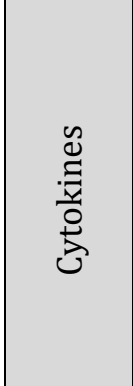 & 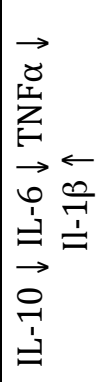 & 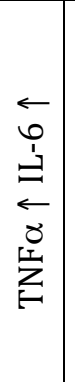 & 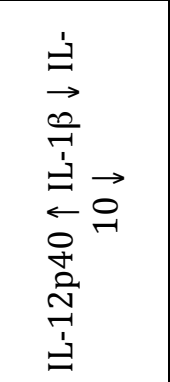 \\
\hline 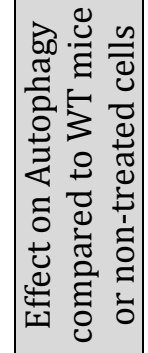 & 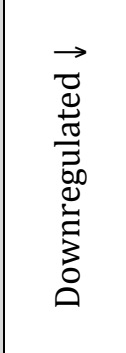 & \multicolumn{3}{|c|}{ 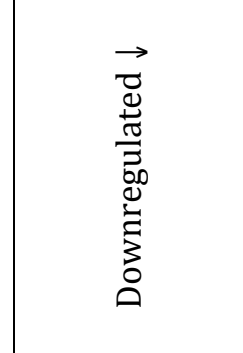 } & 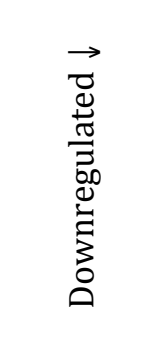 & 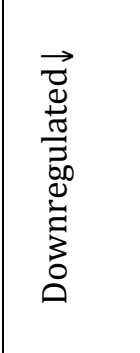 & 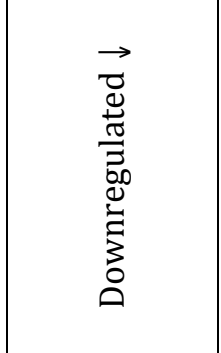 & 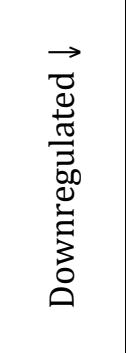 & 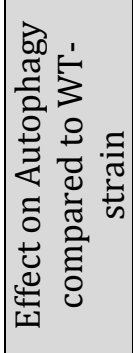 & 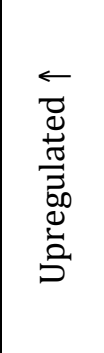 & 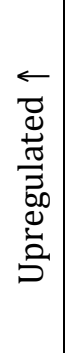 & 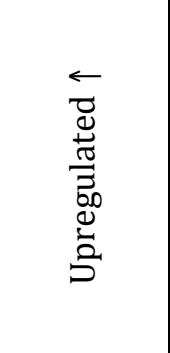 \\
\hline 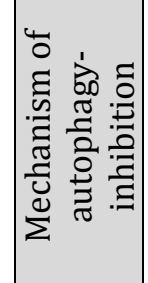 & 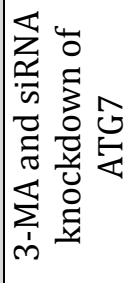 & 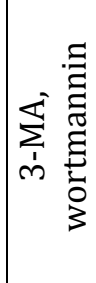 & 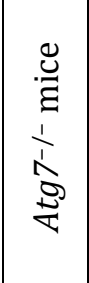 & 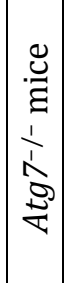 & 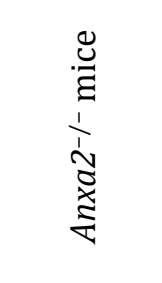 & 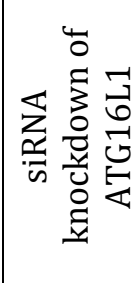 & 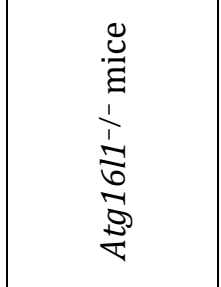 & 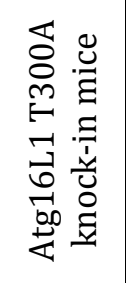 & 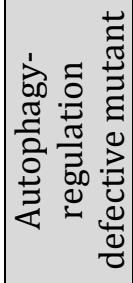 & $\begin{array}{l}\text { ऽै } \\
\stackrel{\bigotimes}{\triangleleft}\end{array}$ & $\ddot{\ddot{d}}$ & $\underset{\vec{\nabla}}{\stackrel{\pi}{\Delta}}$ \\
\hline 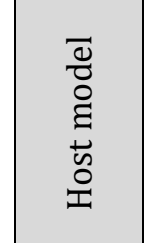 & 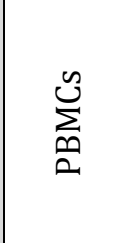 & 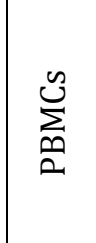 & 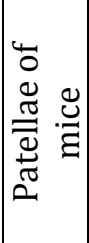 & $\sum_{\infty}^{\infty}$ & 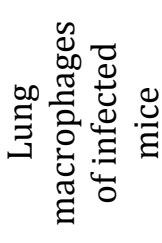 & 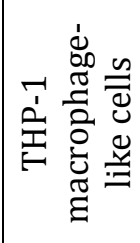 & 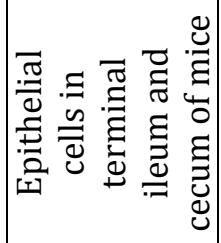 & 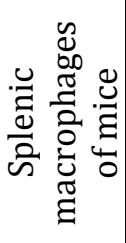 & 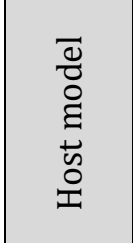 & $\sum_{\infty}^{\infty}$ & $\sum_{n=1}^{n}$ & 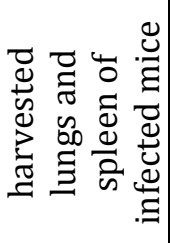 \\
\hline 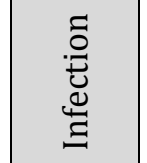 & $\begin{array}{l}\text { D } \\
\frac{\vec{J}}{3} \\
\text { I }\end{array}$ & 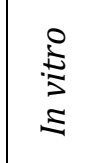 & $\begin{array}{l}\stackrel{0}{\grave{z}} \\
\mathbf{s}\end{array}$ & $\begin{array}{l}0 \\
\frac{D}{3} \\
\Xi\end{array}$ & 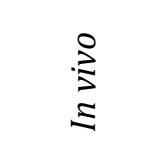 & $\begin{array}{l}\stackrel{0}{ \pm} \\
\stackrel{2}{\Xi} \\
\Xi\end{array}$ & $\begin{array}{l}\stackrel{0}{\grave{2}} \\
\leq\end{array}$ & $\begin{array}{l}\stackrel{0}{ \pm} \\
\stackrel{2}{\Xi} \\
\Xi\end{array}$ & 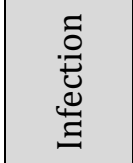 & $\begin{array}{l}\text { D } \\
\frac{ \pm}{2} \\
\text { I }\end{array}$ & $\begin{array}{l}0 \\
\frac{D}{3} \\
\text { I }\end{array}$ & $\begin{array}{l}\stackrel{0}{\grave{z}} \\
\mathbf{I}\end{array}$ \\
\hline $\begin{array}{l}\frac{\pi}{\pi} \\
\frac{\tilde{U}}{\tilde{\pi}} \\
\infty\end{array}$ & 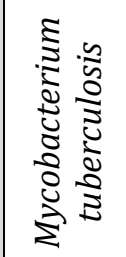 & & $\begin{array}{l}\frac{1}{2} \\
\frac{2}{2} \\
\frac{2}{2} \\
0 \\
0 \\
0 \\
0 \\
0 \\
0\end{array}$ & & 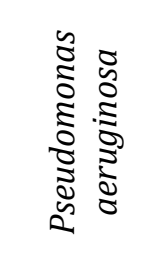 & 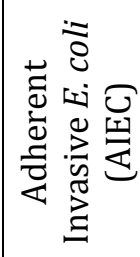 & 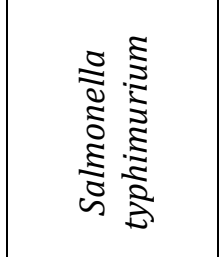 & 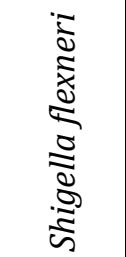 & 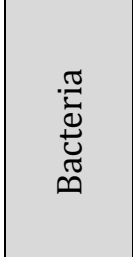 & 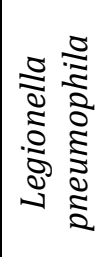 & & 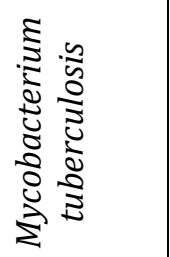 \\
\hline
\end{tabular}




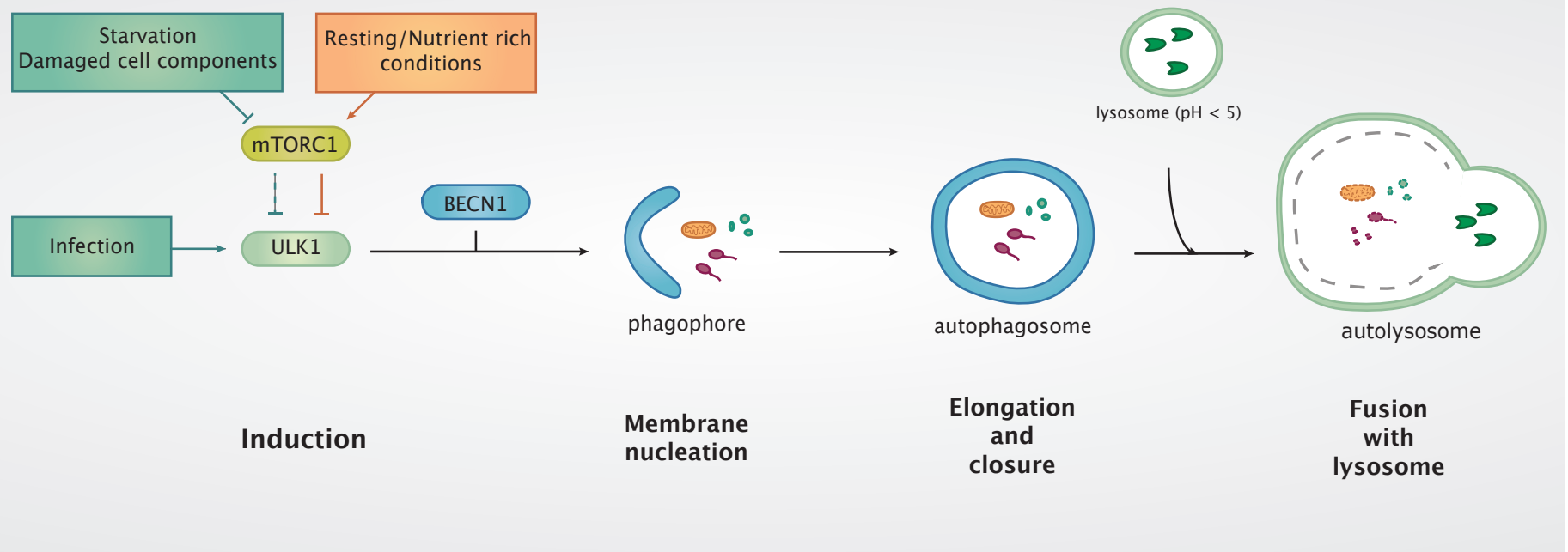




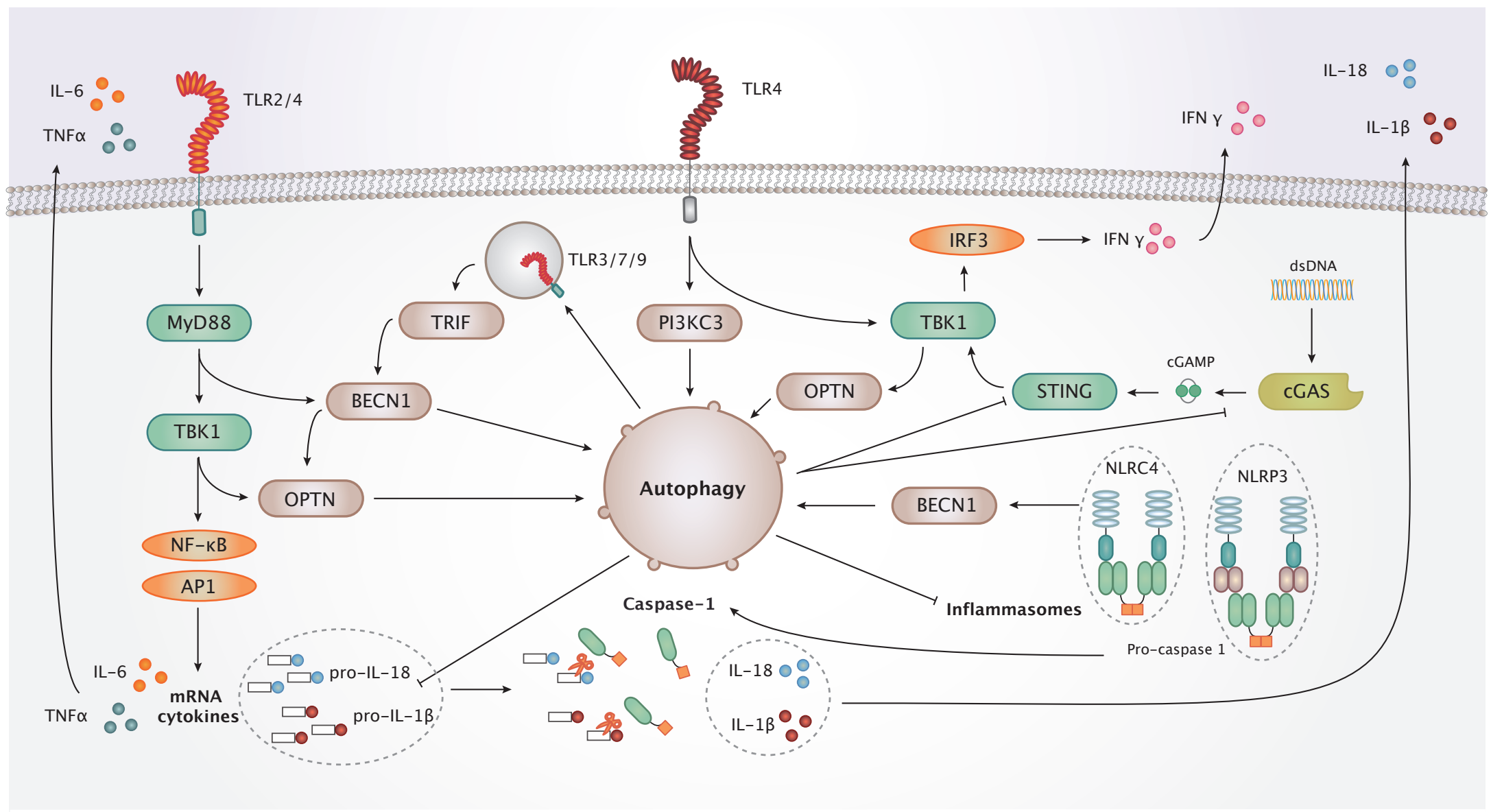

